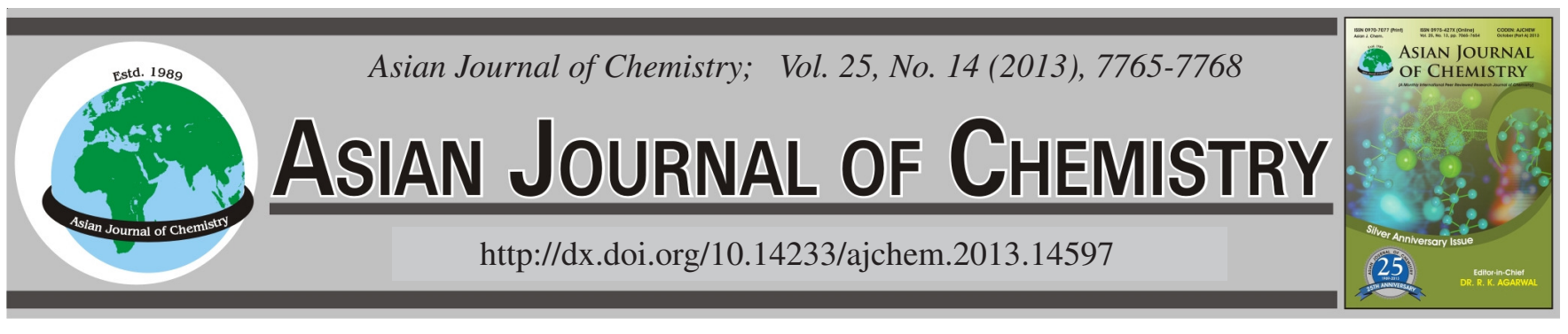

\title{
Ultrasonic-Assisted Extraction of Tanshinones from Korean Red Ginseng by Using Amino-Modified Monolithic Cartridge
}

\author{
TAO ZHU ${ }^{1,2}$, HA EUN PARK ${ }^{2}$ and KyUng Ho Row ${ }^{2, *}$
}

${ }^{1}$ School of Chemistry and Chemical Engineering, Tianjin University of Technology, Tianjin 300384, P.R. China ${ }^{2}$ Department of Chemical Engineering, Inha University, 253 Yonghyun-Dong, Nam-Ku, Incheon 402-751, Korea

*Corresponding author: Fax: +82 32 8724046; Tel: +82 32 8607470; E-mail: rowkho@inha.ac.kr

\begin{abstract}
An amino-modified monolithic cartridge was prepared and used as a solid-phase extraction sorbent for the ultrasonic-assisted extraction of tanshinones from Korean red ginseng. After characterizing the material obtained, the SPE-HPLC method with a $\mathrm{C}_{18}$ column (150 mm $\times 4.6 \mathrm{~mm}$ i.d.) was used to further test the performance of the amino-modified monolithic cartridge. Chromatography was performed by reversed-phase high performance liquid chromatography using a $\mathrm{C}_{18}$ column with ultraviolet detection at $254 \mathrm{~nm}$. The mobile phase was an eluting solution consisting of methanol-water $(78 / 22, \mathrm{v} / \mathrm{v})$ at a flow rate of $0.5 \mathrm{~mL} / \mathrm{min}$. A good linear relationship was demonstrated at an analyte concentration of $0.5-100.0 \mu \mathrm{g} / \mathrm{mL}$. The extraction recoveries ranged from 82.9 to $92.8 \%$ and the inter- and intra-day relative standard deviations were $<6.0 \%$. This method is simple, economical and specific and has been used successfully for the extraction of tanshinones from Korean Red Ginseng. In addition, it avoids a tedious pretreatment and provides a fast, economic and reproducible method for assaying trace drugs in complex samples. This cartridge can be used as a potential tool for the extraction of drugs from natural plants.
\end{abstract}

Key Words: Amino-modified monolith, Tanshinones, Korean red ginseng.

\section{INTRODUCTION}

The root of Korean red ginseng is a well-known traditional medicinal herb that is commonly used for the treatment of a range of diseases ${ }^{1}$, particularly for cardiovascular diseases including coronary heart disease, hypertension and chronic heart failure ${ }^{2,3}$. Korean red ginseng contains abietane-type diterpenes (tanshinones) $)^{4-6}$, such as tanshinone I (TI), tanshinone IIA (TIIA) and cryptotanshinone (CT), (Fig. 1). They exhibited broad-spectrum bactericidal, dilating coronary artery and anticancer effects ${ }^{7}$.
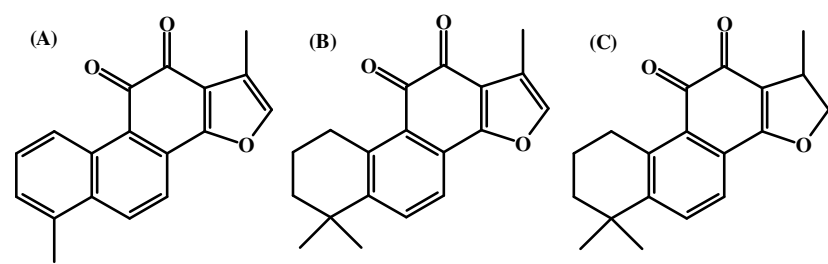

Fig. 1. Molecular structure of tanshinones: (A) TI, (B) TIIA and (C) CT

The traditional extraction of tanshinones from Korean red ginseng by organic solvents has been reported with subsequent analysis using HPLC ${ }^{8-11}$. On the other hand, it is important to remove impurities from the organic solvent extracts. Solid- phase extraction is a viable alternative to the conventional sample preparation methods and it has been applied to natural plant extraction $^{12,13}$. A clean-up stage in the SPE protocol is necessary to enhance the selectivity of the sorbent ${ }^{14}$ and the choice of sorbent is a key point in SPE because it can control the analytical parameters, such as selectivity, affinity and capacity ${ }^{15,16}$.

Many studies have emphasized the importance of improving different stationary phase materials with a range of structures to improve the efficiency and selectivity ${ }^{17}$. A monolithic column has recently become a rapidly interesting field in the preparation of chromatographic stationary phase ${ }^{18,19}$. This column exploits the advantage of low cost, easy preparation and the excellent properties for the extraction of drugs compared to a conventional packing extraction column ${ }^{20,21}$.

In this study, an amino-modified monolithic cartridge was prepared by in situ polymerization. After characterizing the obtained material, a new SPE-HPLC method was developed for the ultrasonic-assisted extraction of tanshinones from Korean red ginseng using this monolithic cartridge.

\section{EXPERIMENTAL}

Korean red ginseng was obtained from a local market (Incheon, Korea). Cryptotanshinone (CT), tanshinone I (TI) 
and tanshinone IIA (TIIA) were purchased from the National Institute for the Control of Pharmaceutical and Biological Products (Beijing, China). Methacrylic acid, glycidyl methacrylate and acrylamide were obtained from Sigma (St Louis, MO, U.S.A.). Ethylene glycol dimethacrylate was acquired from Fluka (Buchs, Switzerland). Dodecanol was purchased from Acros organics (New Jersey, U.S.A.). Cyclohexanol and 2, 2'-azobis (isobutyronitrile) (AIBN) were supplied by Junsei Chemical Co. Ltd., (Japan) and refined prior to use. Methanol, ethanol, acetonitrile and chloroform were purchased from Duksan Pure Chemical Co. Ltd., (Korea). Distilled water (18.2 $\mathrm{M} \Omega \mathrm{cm}$ ) was filtered through a vacuum pump (Division of Millipore, Waters, U.S.A.) and filter (HA-0.45, Division of Millipore, Waters, U.S.A.) prior to use.

The chromatography system consisted of a Waters 600s Multi solvent Delivery System, Waters 616 liquid chromatography (Waters Associates, Milford, MA, U.S.A.), Rheodyne injector ( $20 \mu \mathrm{L}$ sample loop) and a variable wavelength 2487 UV dual channel detector. Autochro-2000 software (Younglin Co. Ltd., Korea) was used as the data acquisition system. All samples were filtered through a filter (MFS-25, $0.2 \mu \mathrm{m}$ TF, WHATMAN, U.S.A.) before injection into the HPLC system.

HPLC analysis was performed on an OptimaPak $\mathrm{C}_{18}$ column $(5 \mu \mathrm{m}, 150 \times 4.6 \mathrm{~mm}$, i.d., RS tech Corporation, Daejeon, Korea) with a guard column $(10 \times 4.6 \mathrm{~mm})$ packed with $\mathrm{C}_{18}$. The mobile phase was methanol/water (78/22, v/v) at a flow rate of $0.5 \mathrm{~mL} \mathrm{~min}^{-1}$ with ultraviolet detection at 254 nm.

Standard sample preparation: The stock solutions of CT, TI and TIIA were prepared by dissolving the appropriate amount of drug in methanol to a concentration of $1,000.0 \mu \mathrm{g} \mathrm{mL}^{-1}$. The working standard solutions of CT, TI and TIIA (0.5-100.0 $\mu \mathrm{g}$ $\mathrm{mL}^{-1}$ ) were prepared by gradual dilutions with methanol.

Preparation of the amino-modified monolithic cartridge: The monolithic column was prepared by in situ polymerization. First, a mixture consisting of $0.5 \mathrm{~mL}$ of methacrylic acid, $1.8 \mathrm{~mL}$ of glycidyl methacrylate, $2.0 \mathrm{~mL}$ of ethylene glycol dimethacrylate, $3.0 \mathrm{~mL}$ of dodecanol, $2.5 \mathrm{~mL}$ of cyclohexanol, $2.5 \mathrm{~mL}$ of methanol and $0.05 \mathrm{~g}$ of AIBN were purged with nitrogen for $15 \mathrm{~min}$. A stainless steel column (100 $\times 9.0 \mathrm{~mm}$, i.d.) sealed at the bottom was filled with the polymerization mixture and then sealed at the top. After polymerization at $55^{\circ} \mathrm{C}$ for $24 \mathrm{~h}$, the column was flushed with methanol to remove the porogen and other soluble compounds. A methanol solution containing $10 \%$ acrylamide was pumped through the column at $0.1 \mathrm{~mL} / \mathrm{min}$ in steps at $80{ }^{\circ} \mathrm{C}$ for $24 \mathrm{~h}$. Finally, the column was washed with $0.05 \mathrm{~mol} / \mathrm{L}$ acetate buffer followed by deionized water until the eluent was neutral.

The obtained monolithic column was dried at $55^{\circ} \mathrm{C}$ for $24 \mathrm{~h}$ and cut into small pieces $(10 \times 9.0 \mathrm{~mm}$, i.d. $)$. The small column core was then placed into a plastic cartridge $(75 \times 9.0$ $\mathrm{mm}$, i.d.) for the SPE process.

Morphology analysis: After the chromatographic experiments, the amino-modified monolithic column was washed with methanol for $60.0 \mathrm{~min}$. Then, the column core was dried, ground and sieved to small particles for the characterization experiment. The microstructure of this material was observed by field emission-scanning electron microscopy (FE-SEM, S-4200 model, Hitachi, Japan) at a voltage of $15 \mathrm{kV}$.
Extraction process: The Korean red ginseng roots were oven dried, sliced and crushed. Typical solvent extraction was carried out by placing $1.0 \mathrm{~g}$ of Korean red ginseng powders into $20 \mathrm{~mL}$ of five different solvents (water, methanol, methanol with $5 \%$ acetic acid solution, ethanol and ethanol with $5 \%$ acetic acid solution) with ultrasonic-assisted extraction. Subsequently, $0.2 \mathrm{~mL}$ of the obtained extraction solution was loaded into a SPE monolithic cartridge and washed with $2.0 \mathrm{~mL}$ of water. Elution was achieved with $2.0 \mathrm{~mL}$ of a methanol solution containing $10 \%$ acetic acid. The filtrate was evaporated to dryness and reconstituted in $0.2 \mathrm{~mL}$ of the mobile phase for further HPLC analysis. Moreover, the monolithic cartridges were washed again with methanol for subsequent use.

\section{RESULTS AND DISCUSSION}

FE-SEM was used to observe the morphology of the aminomodified monolithic polymer, which is an important parameter for evaluating the polymerization stability and reproducibility. Fig. 2 presents a typical FE-SEM image showing the interconnected textural pores and flow-through channels inlaid in the network skeleton of this monolithic polymer. This pore structure was created by a variety of porogen solvents (dodecanol, cyclohexanol and methanol). These pores and channels allowed the mobile phase to flow through the monolith at higher flow rates with low flow resistance. The existence of interconnected pores and high surface area commonly indicates high specific surface areas of the monolithic polymer.

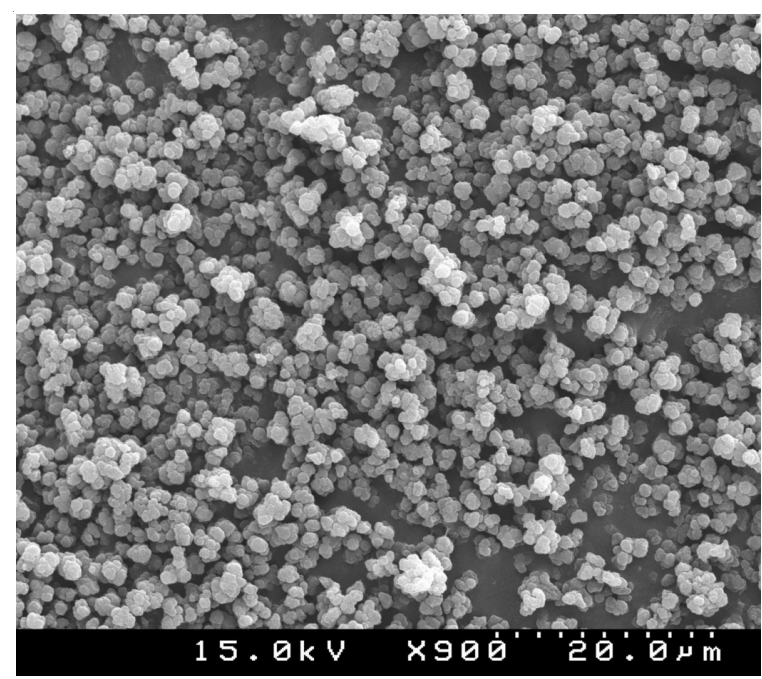

Fig. 2. FE-SEM images of amino-modified monolithic column

\section{Optimization of extraction process}

Effect of extraction solvent: The optimum extraction conditions were studied systematically. A series of extraction solvents, such as water, methanol, methanol solution containing $5 \%$ acetic acid, ethanol and ethanol solution containing $5 \%$ acetic acid, were investigated to achieve the higher extraction yields of tanshinones from Korean red ginseng (Fig. 3). The figure showed the highest extraction yield of tanshinones was obtained when a methanol solution containing $5 \%$ acetic acid solution was used as the extraction solvent. This indicates that the weak acidic solvent was more suitable for extracting tanshinones from Korean red ginseng. 


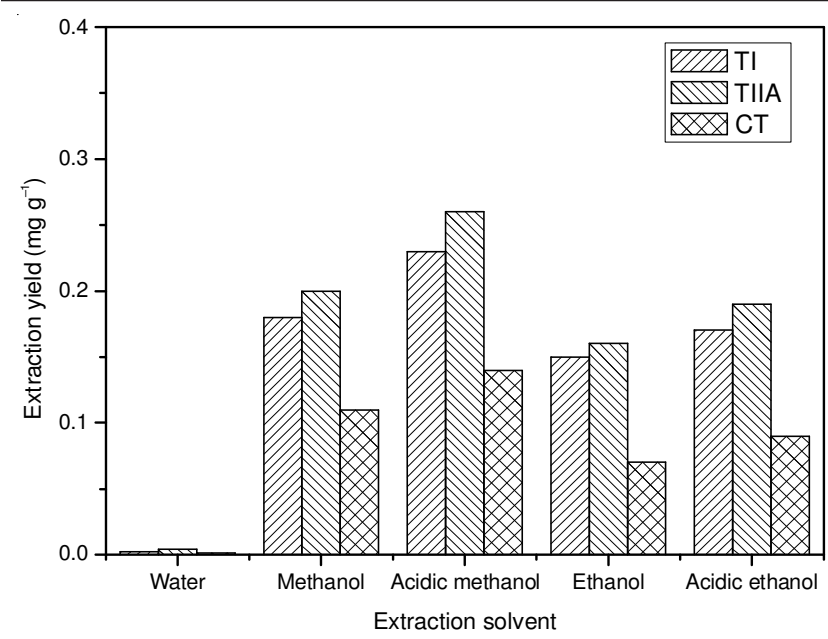

Fig. 3. Effect of different extraction solvents on extraction yields of tanshinones (solid/liquid ratio $\left(\mathrm{g} \mathrm{mL}^{-1}\right)=1 / 20$, ultrasonic power $=$ $100 \mathrm{~W}$, extraction time $=1 \mathrm{~h}$ and extraction temperature $=25^{\circ} \mathrm{C}$ )

Effect of extraction time: The ultrasonic-assisted extraction time affected the yield of tanshinones from Korean red ginseng. Therefore, the effect of the extraction time was investigated from 15-80 min. An extraction time of $60 \mathrm{~min}$ was selected for further extraction after considering the extraction efficiency and to save time.

Optimization of the SPE process: A washing step should be applied immediately after loading the extract from natural plants on the sorbent because it can reduce the matrix interference in the separation of the target. First, water was selected as the washing solvent. To determine the minimum volume of washing solvent, different volumes ranging from 1 to $5 \mathrm{~mL}$ were investigated. The amount of eluted interference increased with increasing volume of water to $2 \mathrm{~mL}$. No more interference was observed when the volume of washing solvent was $>2 \mathrm{~mL}$. Therefore, $2 \mathrm{~mL}$ was considered to be the optimum volume of water. Some interactions were observed between the three target compounds and amino-modified monolithic polymer. A series of elution solutions, such as methanol, methanol with $10 \%$ acetic acid solution, acetonitrile and acetonitrile with $10 \%$ acetic acid solution, were investigated to obtain the higher recovery of tanshinones from Korean red ginseng. A large amount of tanshinones were eluted out from the monolithic cartridge with $2 \mathrm{~mL}$ of a methanol solution containing $10 \%$ acetic acid.

Under the optimized conditions, the proposed SPE-HPLC method was applied to the extraction and purification of tanshinones from Korean red ginseng. Fig. 4A shows the typical HPLC chromatogram without SPE and Fig. 4B presents the result of the SPE extract samples using the amino-modified monolith. The matrix substances were obviously decreased by the monolith and the purification of the three targets was completed with high recovery. The mean extraction yields were obtained; $0.23,0.26$ and $0.14 \mathrm{mg} \mathrm{g}^{-1}$ for TI, TIIA and CT, respectively.

Validation of the proposed analytical method: The calibration curves were constructed using the areas of the chromatographic peaks measured at nine increasing tanshinones concentrations, ranging from 0.5 to $100.0 \mu \mathrm{g} \mathrm{mL} \mathrm{m}^{-1}$. Good linearity was obtained throughout the concentration range and the linear correlation equations were $\mathrm{y}=192.6 \mathrm{x}-27.3\left(\mathrm{r}^{2}=\right.$ $0.9996)$ for TI, $y=212.4 x-109.4\left(r^{2}=0.9998\right)$ for TIIA and $y=177.2 x-97.3\left(r^{2}=0.9996\right)$ for CT. The limit of detection and limit of quantification of the three targets, which are the amounts for which the signal-to-noise- ratios were 3:1 and $10: 1$, respectively, were $0.10 \mu \mathrm{g} \mathrm{mL}{ }^{-1}$ and $0.30 \mu \mathrm{g} \mathrm{mL}$, respectively.

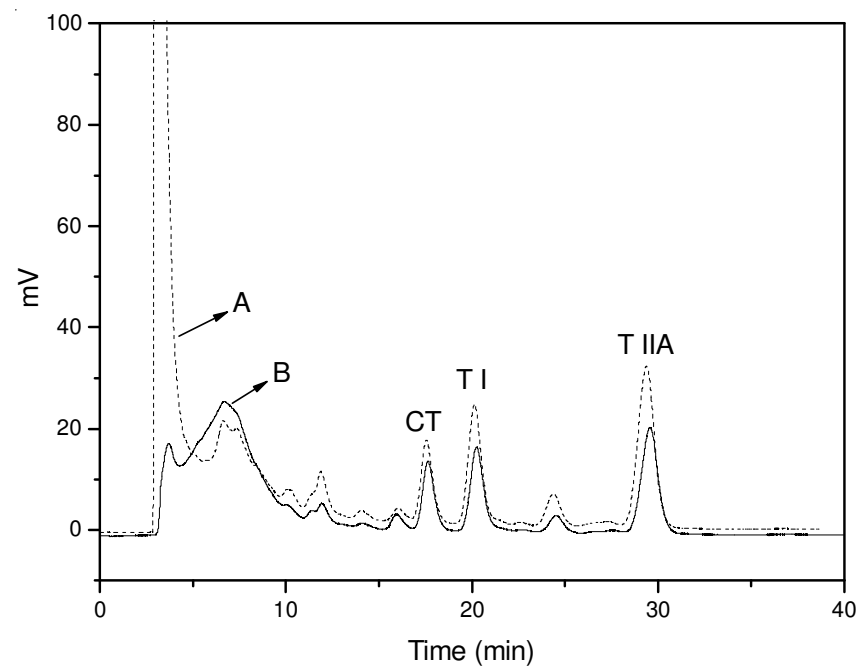

Fig. 4. Chromatograms of acidic methanol extracts from Korean red ginseng on $\mathrm{a}_{18}$ column $(5 \mathrm{~mm}, 150.0 \times 4.6 \mathrm{~mm}$, i.d.) (A. without SPE; B. SPE by amino-modified monolithic cartridge) (Isocratic mobile phase composition was methanol-water $(78: 22, \mathrm{v} / \mathrm{v})$ and the flow rate was $0.5 \mathrm{~mL} \mathrm{~min}^{-1}$ with $\mathrm{UV}$ detection at $254 \mathrm{~nm}$ )

The intra-day and the inter-day precision of the method evaluated as the relative standard deviations ranged from 2.9 to $5.2 \%$. The extraction recoveries of tanshinones from Korean red ginseng were evaluated by spiking three different levels $\left(1,10\right.$ and $\left.50 \mu \mathrm{g} \mathrm{mL}^{-1}\right)$ of the standard analytes to the extract samples in three replicates. The mean recoveries of these three targets (TI, TIIA and CT) were 82.9 to $88.6,84.7$ to 90.3 and 91.2 to $92.8 \%$, respectively, confirming the reliability of this method (Table-1).

\section{TABLE-1}

INTRA-DAY AND INTER-DAY PRECISIONS AND RECOVERY STUDIES OF TI, TIIA AND CT IN KOREAN RED GINSENG $(n=3)$

\begin{tabular}{ccc|cc} 
& \multicolumn{2}{c|}{$\begin{array}{c}\text { RSD (\%) inter-day } \\
\text { RSD (\%) }\end{array}$} & \multicolumn{2}{c}{ Recovery } \\
\cline { 2 - 5 } & Intra-day & Inter-day & $\begin{array}{c}\text { Amount added } \\
(\mu \mathrm{g} / \mathrm{mL})\end{array}$ & $\begin{array}{c}\text { Average } \\
\text { recovery (\%) }\end{array}$ \\
\hline \multirow{3}{*}{ T I } & 4.8 & 5.2 & 1.0 & 88.6 \\
& 2.6 & 2.9 & 10.0 & 84.3 \\
& 3.7 & 4.8 & 50.0 & 82.9 \\
\hline \multirow{3}{*}{ T IIA } & 2.9 & 4.9 & 1.0 & 85.3 \\
& 4.3 & 4.8 & 10.0 & 84.7 \\
& 3.8 & 3.6 & 50.0 & 90.3 \\
\hline \multirow{2}{*}{ CT } & 3.3 & 4.6 & 1.0 & 91.2 \\
& 3.8 & 4.0 & 10.0 & 92.5 \\
& 2.6 & 3.5 & 50.0 & 92.8 \\
\hline
\end{tabular}

\section{Conclusion}

A new amino-modified monolithic cartridge was synthesized and evaluated by FE-SEM. An SPE-HPLC method was developed for the purification of tanshinones from Korean red 
ginseng and the performance of the amino-modified monolith was confirmed. Under optimized conditions, good linearity was obtained with RSDs $<4.8 \%$ and extraction recoveries of 82.9-92.8\%. The extraction yields for tanshinone I, tanshinone IIA and cryptotanshinone were $0.23,0.26$ and $0.14 \mathrm{mg} \mathrm{g}^{-1}$, respectively. The developed method showed high sensitivity as well as suitable precision, accuracy and recovery. This type of hybrid monolith allows the separation and purification of bioactive compounds from natural plant extracts. Overall, the purposed method has many advantages, such as low cost, simple procedure and good stability, compared to a previous study of the extraction of tanshinones using ionic liquidmodified silica sorbents.

\section{ACKNOWLEDGEMENTS}

This research was supported by the Basic Science Research Program through the National Research Foundation of Korea (NRF) funded by the Ministry of Education, Science and Technology (2012-0005250).

\section{REFERENCES}

1. Z. Shi, J. He and W. Chang, Talanta, 64, 401 (2004).

2. H.W. Luo, B.J. Wu, M.Y. Wu and Z.G. Yong, Phytochemistry, 24, 815 (1985).
3. H.Y. Zhang, Z.D. Hu, G.L. Yang and H.W. Sun, Chromatographia, 49, 219 (1999).

4. H.T. Trinh, S.J. Chae, E.H. Joh, K.H. Son, S.J. Jeon and D.H. Kim, J. Ethnopharmacol., 132, 344 (2010).

5. M.A. Mosaddik, Phytomedicine, 10, 682 (2003).

6. X. Wang, W.Y.W. Lee, P.M.Y. Or and J.H.K. Yeung, Phytomedicine, 16, 712 (2009).

7. X. Pan, G. Niu and H. Liu, Biochem. Eng. J., 12, 71 (2002).

8. N. Okamura, K. Kobayashi, A. Yagi, T. Kitazawa and K. Shimomura, J. Chromatogr. A, 542, 317 (1991).

9. J.R. Dean, B. Liu and R. Price, J. Chromatogr. A, 799, 343 (1998).

10. X. Pan, G. Niu and H. Liu, J. Chromatogr. A, 922, 371 (2001).

11. Z. Shi, J. He, T. Yao, W. Chang and M. Zhao, J. Pharm. Biomed. Anal., 37, 481 (2005).

12. T. Zhu and K.H. Row, Chromatographia, 69, 1477 (2009).

13. T. Zhu, C.H. Yoon and K.H. Row, Chin. J. Chem., 29, 1246 (2011).

14. Z. Li, X. Chang, X. Zou, X. Zhu, R. Nie, Z. Hu and R. Li, Anal. Chim. Acta, 632, 272 (2009).

15. T. Zhu and K.H. Row, J. Liq. Chromatogr. Rel. Technol., 32, 1423 (2009).

16. Y.Q. Cai, G.B. Jiang, J.F. Liu and Q.X. Zhou, Anal. Chem., 75, 2517 (2003).

17. T. Zhu, W. Bi and K.H. Row, J. Appl. Polym. Sci., 118, 3425 (2010).

18. L. Bai, H. Liu, Y. Liu, X. Zhang, G. Yang and Z. Ma, J. Chromatogr. A, 1218, 100 (2011).

19. G. Yang, H. Liu, L. Bai, M. Jiang and T. Zhu, Micropor. Mesopor. Mater, 112, 351 (2008).

20. T. Zhu and K.H. Row, Chromatographia, 71, 981 (2010).

21. T. Zhu, S. Li and K.H. Row, J. Appl. Polym. Sci., 121, 1691 (2011). 